\title{
A Torus Bifurcation Theorem with Symmetry
}

\author{
S. A. van Gils ${ }^{1}$ and M. Golubitsky ${ }^{2}$
}

Received December 27, 1988

\begin{abstract}
A general theory for the study of degenerate Hopf bifurcation in the presence of symmetry has been carried out only in situations where the normal form equations decouple into phase/amplitude equations. In this paper we prove a theorem showing that in general we expect such degeneracies to lead to secondary torus bifurcations. We then apply this theorem to the case of degenerate Hopf bifurcation with triangular $\left(D_{3}\right)$ symmetry, proving that in codimension two there exist regions of parameter space where two branches of asymptotically stable 2-tori coexist but where no stable periodic solutions are present. Although this study does not lead to a theory for degenerate Hopf bifurcations in the presence of symmetry, it does present examples that would have to be accounted for by any such general theory.
\end{abstract}

KEY WORDS: torus; Hopf bifurcation; symmetry.

\section{INTRODUCTION}

One of the more interesting features of degenerate Hopf bifurcations in the presence of symmetry is the appearance, via secondary bifurcation, of quasiperiodic motion on a torus. In this paper we concentrate on twoparameter systems of ODE and prove theorems that allow us to find and compute the direction of branching for some of these tori. The advantage of our approach is that we determine this information using only the Taylor expansion of the vector field at the point where degenerate Hopf bifurcation occurs.

The simplest form of Hopf bifurcation with symmetry group $\Gamma$ occurs as follows. We assume that $\Gamma$ is a compact Lie group acting absolutely

\footnotetext{
${ }^{1}$ Department of Applied Mathematics, Universiteit Twente, P.O. Box 217, 7500 AE, Enschede, The Netherlands.

${ }^{2}$ Department of Mathematics, University of Houston, Houston, Texas 77204-3476.
} 
irreducibly on a vector space $V$, that is, the only matrices on $V$ which commute with $\Gamma$ are multiples of the identity.

Let

$$
d z / d t=f(z, \lambda), \quad z \in V \oplus V=V \otimes \mathbb{C}
$$

be a system of ODE where $f$ is $\Gamma$-equivariant. In complex coordinates, absolute irreducibility implies that

$$
f(z, \lambda)=a(\lambda) z+\cdots
$$

where $a(\lambda) \in \mathbb{C}$. We say that (1.1) has a Hopf bifurcation at $\lambda=0$ if $a(0)$ is purely imaginary.

The standard Hopf theorem $(V=\mathbb{R}, \Gamma=1)$ states that if the eigenvalue crossing condition

$$
\frac{d}{d \lambda} \operatorname{Re}(a)(0) \neq 0
$$

holds, then there exists a unique branch of periodic solutions to (1.1). Moreover, if a certain coefficient $\mu_{2}$ involving the second- and third-order terms in $f$ satisfies

$$
\mu_{2} \neq 0
$$

then the direction of branching (supercritical or subcritical) and the asymptotic stability of these periodic solutions are determined. We call a Hopf bifurcation degenerate if either (1.3) or (1.4) fails. Such singularities are studied by Takens (1974) and Golubitsky and Langford (1981).

In Hopf bifurcation with symmetry we have a degeneracy if either the direction of branching or the asymptotic stability of a branch of periodic solutions is not determined at the lowest order that it could have been. We are interested in such degeneracies because they may be unavoidable in two parameter systems. Degenerate Hopf bifurcations with $O(2)$-symmetry have been studied extensively for the past few years and the results concerning this specific case are discussed in Section 2.

We now explain why one should expect invariant tori to be produced by perturbing certain of these degeneracies. To do this we recall some of the theory of Hopf bifurcation with symmetry. We assume that $f$ is in Birkhoff normal form, that is, we assume that

$$
f \text { is } \Gamma \times S^{1} \text {-equivariant }
$$

where for $x \otimes c \in V \otimes \mathbb{C}$ we have $(\gamma, \theta)(x \otimes c)=(\gamma x) \otimes\left(e^{i \theta} c\right)$. The $S^{1}$ 
symmetry comes from phase shifts. We detect branches of periodic solutions by choosing a subgroup $\Sigma \subset \Gamma \times S^{1}$ such that

$$
\operatorname{dim} \operatorname{Fix}(\Sigma)=2
$$

where

$$
\operatorname{Fix}(\Sigma)=\{z \in V \otimes \mathbb{C}: \sigma z=z, \forall \sigma \in \Sigma\}
$$

In normal form $f: \operatorname{Fix}(\Sigma) \times \mathbb{R} \rightarrow \operatorname{Fix}(\Sigma)$. Thus, the differential equation (1.1) restricted to $\operatorname{Fix}(\Sigma)$ satisfies the hypotheses of standard Hopf bifurcation. In particular, if (1.3) and (1.4) hold for the restricted system, then there exists a branch of periodic solutions for $(1.1)$ in $\operatorname{Fix}(\Sigma)$ and the direction of branching (in $\lambda$ ) is determined. [Golubitsky and Stewart (1985) show that the assumption that $f$ is in Birkhoff normal formal is not needed to prove these points.]

Stability of these solutions, however, is not determined by the standard Hopf theorem, since the Floquet multipliers corresponding to eigenvectors in $\mathrm{Fix}(\Sigma)^{\perp}$ also enter into this determination of stability. In this paper we focus on degeneracies produced when determining stability along known branches of periodic solutions obtained using (1.6). Specifically, suppose that one tracks along a branch of periodic solutions and that at some special value $\lambda=\lambda_{0}$ the periodic solution loses stability by having a simple complex conjugate pair of Floquet multipliers cross through the unit circle with nonzero speed. The torus bifurcation theorem (see Iooss, 1979) guarantees the existence of invariant tori. Suppose now that (1.1) depends on a second parameter $\mu$ and that the critical value $\lambda_{0}$ also depends on $\mu$. We can imagine a situation where as $\mu$ is varied $\lambda_{0}$ moves into the origin, say at $\mu=0$. When this happens we will find a degenerate Hopf bifurcation with symmetry. Moreover, it seems reasonable that the speed of the Floquet exponent that crosses through zero and the direction of branching of the branch for tori can be determined from the Taylor expansion of $f$ at the origin and with $\lambda$ and $\mu$ set to zero. Our results are summarized in Theorems 4.5 and 4.6.

It is important to understand what the effect of the $S^{1}$ symmetry is, that is, what will happen when this symmetry is broken at high order. In this respect it is notable that the two frequencies of the 2-torus are of different orders of magnitude. This disparity often occurs in resonance problems and here, due to spatial symmetry, we have $1: 1$ resonance. Hence a quotient of the frequencies will vanish at $\lambda=\mu=0$. Nevertheless, generically we can independently control the variation of these frequencies. Although standard theorems of dissipative KAM theory do not apply directly, this control seems sufficient to conclude that invariant 2-tori will persist on a Cantor set of large measure. See van Gils et al. (1989). 
We note that several authors have considered the bifurcation of tori from branches of periodic solutions. See Renardy (1982), Rand (1982), and Ruelle (1973). An important point here is that the Floquet matrix itself commutes with the isotropy subgroup $\Sigma$, and as a result the Floquet multipliers may be forced by symmetry to have high multiplicity. See Chossat and Golubitsky (1988). Thus the assumption above that the Floquet multipliers are simple may not always be satisfied.

The scenario that we described above does happen in the case of $O(2)$ symmetry. However, as we explain in Section 2, there is a relatively simple way to analyze the resulting tori (the torus bifurcation theorem is not needed there). In addition, the resulting flow on the torus is particularly simple. Symmetry forces the flow to be linear.

A more interesting example occurs in Hopf bifurcation with $D_{n}$ symmetry. Here the generic Hopf theory has been worked out (Golubitsky and Stewart, 1986; van Gils and Valkering, 1986). Because in this case $\operatorname{dim} V=2$, it follows that $\operatorname{dim} \operatorname{Fix}(\Sigma)^{\perp}=2$ and the Floquet multipliers discussed above must be simple. It is this example (itself motivated by considering rings of oscillators) that has motivated our theorem. In Section 3 we discuss the general results for Hopf bifurcation with $D_{n}$ symmetry, while in Section 5 we illustrate our theorem by explicitly calculating the direction of branching of tori in the $D_{3}$ case. Bifurcation diagrams corresponding to degenerate Hopf bifurcation with $D_{3}$ symmetry are presented in Section 6 .

In Section 4 we present our hypotheses and theorems. This section can be read directly after the Introduction since explicit knowledge of the $O(2)$ and $D_{n}$ examples is not needed for the general theory. The general theorem, however, is obtained by abstracting aspects of these $D_{n}$ examples. In Section 5 we show how to find two-frequency motions by applying standard Hopf bifurcation results to a certain normal vector field whose existence is determined in Section 4.

\section{DEGENERATE HOPF BIFURCATION WITH $O$ (2) SYMMETRY}

We begin by surveying some of the results on degenerate Hopf bifurcations with $O(2)$ symmetry. This problem has been studied by Erneux and Matkowsky (1984), Knobloch (1986), Chossat (1986), Golubitsky and Roberts (1987), Nagata (1986), and Crawford and Knobloch (1988).

The action of $O(2)$ on $\mathbb{R}^{4}=\mathbb{C} \oplus \mathbb{C}$ is generated by

$$
\begin{aligned}
& \theta\left(z_{1}, z_{2}\right)=\left(e^{i \theta} z_{1}, e^{i \theta} z_{2}\right), \quad \forall \theta \in S O(2) \\
& \kappa\left(z_{1}, z_{2}\right)=\left(\bar{z}_{1}, \bar{z}_{2}\right)
\end{aligned}
$$


Consider the $O(2)$-equivariant system of $\mathrm{ODE}$

$$
\frac{d z}{d t}=f(z, \lambda), \quad f(0, \lambda)=0
$$

depending on a bifurcation parameter $\lambda$. We assume that (2.2) has a Hopf bifurcation at $\lambda=0$; due to symmetry the eigenvalues $\sigma(\lambda) \pm i \omega(\lambda)$ of $(d f)_{0, \lambda}$ are each of multiplicity two. By Hopf bifurcation we mean that $\sigma(0)=0$, $\omega(0) \equiv \omega_{0} \neq 0$.

van Gils and Mallet-Paret (1984), Chossat and Iooss (1985), Golubitsky and Stewart (1985), and others have shown that if

$$
\sigma^{\prime}(0) \neq 0
$$

then two branches of periodic solutions $z(t)$ bifurcate from the origin, and moreover, these solutions may be detected by their symmetry. They are

$$
\begin{aligned}
& \text { rotating waves (RW): } \theta z(t)=z(t-\theta) \\
& \text { standing waves (SW): } \kappa z(t)=z(t)
\end{aligned}
$$

Generically, the exchange of stability for such systems goes as follows. Assume that $x=0$ is asymptotically stable when $\lambda<0$. Then for either branch (2.4) to consist of asymptotically stable periodic solutions, both branches must be supercritical, and then precisely one branch consists of stable solutions. More precisely, there are two coefficients, derived from the third-order terms of $f$, which determine the direction of branching of solutions (2.4), with stability being determined by which coefficient is larger.

Erneux and Matkowsky (1984) observed that when such systems depend on two parameters,

$$
\frac{d z}{d t}=f(z, \lambda, \mu)
$$

it is possible to arrange for a distinguished value of $\mu$, say $\mu=0$, where both cubic coefficients are equal. They show that invariant 2 -tori exist in (2.5) for $\mu$ near 0 . The types of bifurcation diagrams which may occur are shown in Fig. 5.3 of Golubitsky and Roberts (1987). [The direction of branching and the stability of the 2-tori depend on fifth- and seventh-order terms in $f$.]

In retrospect the existence of these 2-tori can be understood in a relatively simple way. First, assume that $(2.5)$ is in Birkhoff normal form, which means that now $f$ may be assumed to be $O(2) \times S^{1}$-equivariant (cf. 
Golubitsky and Stewart, 1985). In normal form, (2.5) splits into phaseamplitude equations where the amplitude equations have the form

$$
\frac{d}{d t}\left[\begin{array}{l}
r_{1} \\
r_{2}
\end{array}\right]=\rho\left(r_{1}^{2}+r_{2}^{2}, r_{1}^{2} r_{2}^{2}, \lambda, \mu\right)\left[\begin{array}{l}
r_{1} \\
r_{2}
\end{array}\right]+q\left(r_{1}^{2}+r_{2}^{2}, r_{1}^{2} r_{2}^{2}, \lambda, \mu\right)\left(r_{2}^{2}-r_{1}^{2}\right)\left[\begin{array}{c}
r_{1} \\
-r_{2}
\end{array}\right]
$$

where $r_{j}=\left|z_{j}\right|$. Nontrivial equilibria $\left(r_{1}, r_{2}\right)$ of (2.6) correspond to standing waves $\left(r_{1}=r_{2}\right)$, rotating waves $\left(r_{1} r_{2}=0\right)$, or invariant 2-tori $\left(r_{1} \neq r_{2}\right.$, $r_{1} r_{2} \neq 0$ ).

Thus the Erneux and Matkowsky 2-tori are on the same footing as the periodic solutions in the study of degenerate Hopf bifurcation with $O(2)$ symmetry. Swift (1984) noted that the amplitude equations (2.6) have $D_{4}$-symmetry [generated by $\left(r_{1}, r_{2}\right) \rightarrow\left(r_{1},-r_{2}\right),\left(-r_{1}, r_{2}\right)$ and $\left.\left(r_{2}, r_{1}\right)\right]$. Therefore, degenerate $O(2)$ Hopf bifurcations can be studied using $D_{4^{-}}$ equivariant singularity theory just as degenerate Hopf bifurcation without symmetry can be studied by $Z_{2}$-equivariant singularity theory (see Golubitsky and Langford, 1981). The $D_{4}$-classification was carried out up to (topological) $D_{4}$-codimension two by Golubitsky and Roberts (1987). See also Crawford and Knobloch (1988) or Golubitsky et al. (1988).

It should also be noted that these 2-tori have a special structure due to the $O(2) \times S^{1}$ symmetry of normal form. The flow on the 2-tori is linear. Chossat (1986) has shown that this property persists, even if $f$ is not assumed to be in Birkhoff normal form. His technique is to use a Liapunov-Schmidt reduction to look for two frequency solutions of the form

$$
z(t)=e^{i \omega t} R_{\eta t} q
$$

whjere $R_{\theta}$ is the rotation matrix corresponding to $\theta \in S O(2)$. The function (2.7) has two independent frequencies if $\omega / \eta$ is irrational.

\section{GENERIC HOPF BIFURCATION WITH $D_{n}$ SYMMETRY}

When $n \geqslant 3$ the group $D_{n}$ has two-dimensional irreducible representations. Thus, in systems with $D_{n}$ symmetry, Hopf bifurcation from a $D_{n}$-invariant steady state may occur by eigenvalues of multiplicity one or two crossing the imaginary axis. In this section we review the results of Golubitsky and Stewart (1986) and van Gils and Valkering (1986) about generic $D_{n}$-Hopf bifurcation in the double eigenvalue case. See also Golubitsky et al. (1988). 
Without loss of generality we may assume that the action of $D_{n}$ on $\mathbb{R}^{2} \cong \mathbb{C}$ is generated by

$$
\begin{aligned}
& \gamma \cdot z=e^{i \gamma} z \quad \text { where } \quad \gamma=2 \pi / n \\
& \kappa(z)=\bar{z}
\end{aligned}
$$

It is possible to choose coordinates on $\mathbb{C}^{2}$ such that the action of $D_{n} \times S^{1}$ is generated by

$$
\begin{aligned}
\gamma \cdot\left(z, z_{2}\right) & =\left(e^{i \gamma} z_{1}, e^{i \gamma} z_{2}\right) \\
\kappa \cdot\left(z_{1}, z_{2}\right) & =\left(z_{2}, z_{1}\right) \\
\theta \cdot\left(z_{1}, z_{2}\right) & =\left(e^{i \theta} z_{1}, e^{-i \theta} z_{2}\right)
\end{aligned}
$$

It can be shown that for each $n$, there are precisely three (conjugacy classes of) isotropy subgroups whose fixed point subspaces are two-dimensional. There exists a unique branch of periodic solutions for each of these subgroups in $D_{n}$-symmetric generic Hopf bifurcation. There are a discrete analogue of a "rotating wave" and two discrete analogues of "standing waves."

The rotating wave has isotropy subgroup

$$
\tilde{\mathbb{Z}}_{n}=\left\{(\gamma, \gamma): \gamma \in \mathbb{Z}_{n}\right\}
$$

The standing waves each have isotropy subgroups isomorphic to $\mathbb{Z}_{2}$. The symmetry of one of the standing waves is generated by $\kappa$; the symmetry of the other standing wave is generated by $(\kappa, \pi) \in D_{n} \times S^{1}$, at least when $n \neq 0$ mod 4. See Golubitsky and Stewart (1986) for details.

The exchange of stability for these branches goes as follows. Suppose the $D_{n}$-invariant steady state is stable subcritically and loses stability by having eigenvalues cross the imaginary axis with nonzero speed. When $n \neq 4$, no branch is stable unless all branches are supercritical. There is one third-order term that determines whether the rotating waves are supercritical and another third-order term that determines whether the standing waves are (jointly) supercritical. No branch consists of stable trajectories unless all branches are supercritical, in which case precisely one branch consists of stable solutions.

Supposing that all branches are supercritical, then it can be determined at third order whether the rotating wave or one of the standing waves is stable. If a standing wave is to be stable, then which one is stable is determined by a coefficient of order

$$
m= \begin{cases}n+2 & n \text { odd } \\ (n+2) / 2 & n \text { even }\end{cases}
$$


In Section 5 we show that the conditions for our torus bifurcation theorem (Theorem 4.5) may be satisfied when, in a two-parameter system, there is an isolated value of the parameters where a Hopf bifurcation occurs and the bifurcating branches are neutrally stable at third order.

The remainder of this section is devoted to discussing these results in more detail. The notation we set here is used in Section 5 . We begin by describing the $D_{n} \times S^{1}$ invariants and equivariants.

Recall $m$ as defined in (3.3) and define

$$
\begin{aligned}
& N=\left|z_{1}\right|^{2}+\left|z_{2}\right|^{2} \\
& P=\left|z_{1}\right|^{2}\left|z_{2}\right|^{2} \\
& S=\left(z_{1} \bar{z}_{2}\right)^{m}+\left(\bar{z}_{1} z_{2}\right)^{m} \\
& T=i\left\{\left|z_{1}\right|^{2}-\left|z_{2}\right|^{2}\right\}\left\{\left(z_{1} \bar{z}_{2}\right)^{m}-\left(\bar{z}_{1} z_{2}\right)^{m}\right\}
\end{aligned}
$$

Proposition 3.1. Let $D_{n} \times S^{1}$ act on $\mathbb{C}^{2}$ as defined by (3.2).

(a) Every smooth $D_{n} \times S^{1}$-invariant germ $f: \mathbb{C}^{2} \rightarrow \mathbb{R}$ has the form

$$
f\left(z_{1}, z_{2}\right)=h(N, P, S, T)
$$

for some smooth germ $h: \mathbb{R}^{4} \rightarrow \mathbb{R}$.

(b) Every smooth $D_{n} \times S^{1}$-equivariant germ $g: \mathbb{C}^{2} \rightarrow \mathbb{C}^{2}$ has the form

$$
g=A\left[\begin{array}{l}
z_{1} \\
z_{2}
\end{array}\right]+B\left[\begin{array}{l}
z_{1}^{2} \bar{z}_{1} \\
z_{2}^{2} \bar{z}_{2}
\end{array}\right]+C\left[\begin{array}{l}
\bar{z}_{1}^{m-1} z_{2}^{m} \\
z_{1}^{m} \bar{z}_{2}^{m-1}
\end{array}\right]+D\left[\begin{array}{l}
z_{1}^{m+1} \bar{z}_{2}^{m} \\
\bar{z}_{1}^{m} z_{2}^{m+1}
\end{array} \mid\right.
$$

where $A, B, C, D$ are complex-valued $D_{n} \times S^{1}$-invariant germs.

We consider the system of ODE

$$
\frac{d z}{d t}=g(z, \lambda)
$$

where $g: \mathbb{C}^{2} \times \mathbb{R} \rightarrow \mathbb{C}^{2}$ is $D_{n} \times S^{1}$-equivariant. That is, we assume that we are studying Hopf bifurcation with $D_{n}$-symmetry for a system of ODE which is already in Poincaré-Birkhoff normal form to all orders.

In Table I we present the equations determining the branching of solutions for each of the three isotropy subgroups mentioned above. These results are taken from Golubitsky and Stewart (1986).

The asymptotic stability of these solutions is determined by the signs of the real parts of the eigenvalues of $d g$ evaluated at the solution. The actual computation of these eigenvalues is aided substantially by the 
Table I. Branching Equations for Maximal Isotropy

\begin{tabular}{clc}
\hline Solution type & $\left(z_{1}, z_{2}\right)$ orbit rep. & \multicolumn{1}{c}{ Branching equation } \\
\hline $\begin{array}{l}\text { Rotating wave } \\
\text { Standing wave 1 }\end{array}$ & $(u, 0)$ & $\operatorname{Re}\left(A+B u^{2}\right)=0$ \\
$\begin{array}{c}\text { Standing wave 2 } \\
n \neq 0 \bmod 4\end{array}$ & $(u,-u)$ & $\operatorname{Re}\left(A+B u^{2}+C u^{2 m-2}+D u^{2 m}\right)=0$ \\
$n=0 \bmod 4$ & $\left(u, e^{2 \pi i / n} u\right)$ & \\
\hline
\end{tabular}

existence of the $D_{n} \times S^{1}$ symmetry. In particular, for each of the three isotropy subgroups $\Sigma$, the space $\mathbb{C}^{2}$ may be written as

$$
\operatorname{Fix}(\Sigma) \oplus \operatorname{Fix}(\Sigma)^{\perp}
$$

where $\Sigma$ acts nontrivialiy on $\operatorname{Fix}(\Sigma)^{\perp}$. Since $d g$ evaluated at a solution commutes with $\Sigma$, it follows that both $\operatorname{Fix}(\Sigma)$ and $\operatorname{Fix}(\Sigma)^{\perp}$ are $d g$ invariant subspaces. Thus, the eigenvalues of $d g$ are obtained in each case by finding the eigenvalues of two $2 \times 2$ matrices. Moreover, the $S^{1}$ symmetry forces one eigenvalue of $d g \mid \operatorname{Fix}(\Sigma)$ to have real part zero. Also, for rotating waves when $n \neq 4$, the group $\tilde{Z}_{n}$ acts on $\operatorname{Fix}\left(\tilde{Z}_{n}\right)^{\perp}$ as nontrivial rotations and forces $d g \mid \operatorname{Fix}\left(\tilde{Z}_{n}\right)^{\perp}$ to itself be a scalar multiple of a rotation matrix.

Using this group theoretic information, it is possible to compute the signs of the real parts of the eigenvalues of $d g$. These results are summarized in Table II.

The directions of branching and the asymptotic stability of the branches discussed above follow from Tables I and II assuming that the nondegeneracy conditions

$$
\begin{aligned}
\operatorname{Re}\left(A_{N}+B\right) & \neq 0 \\
\operatorname{Re}(B) & \neq 0 \\
\operatorname{Re}\left(2 A_{N}+B\right) & \neq 0 \\
\operatorname{Re}(B \bar{C}) & \neq 0 \\
\operatorname{Re}\left(A_{\lambda}\right) & \neq 0
\end{aligned}
$$

hold when evaluated at the origin. Observe that these branches are all neutrally stable at third order if

$$
\operatorname{Re} B(0,0)=0
$$

It is this coefficient that may vanish in a two-parameter system and that must be zero in order to aply our torus bifurcation theorem. 
Table II. Signs of Eigenvalues of $d g$ Along Primary Branches

\begin{tabular}{llc}
\hline Solution type & EV of $d g \mid \operatorname{Fix}(\Sigma)$ & EV of $d g \mid \operatorname{Fix}(\Sigma)^{\perp}$ \\
\hline Rotating wave & $-\operatorname{Re}\left(A_{N}+B\right)+O(u)$ & $-\operatorname{Re} B$ \\
Standing wave 1 & $-\operatorname{Re}\left(2 A_{N}+B\right)+O(u)$ & $\operatorname{tr}=\operatorname{Re}\left(B-(m+1) C u^{2 m-4}\right)+O\left(u^{2 m-2}\right)$ \\
& & $\operatorname{det}=-\operatorname{Re}(B \bar{C})+O(u)$ \\
Standing wave 2 & $-\operatorname{Re}\left(2 A_{N}+B\right)+O(u)$ & $\operatorname{tr}=\operatorname{Re}\left(B+(m+1) C u^{2 m-4}\right)+O\left(u^{2 m-2}\right)$ \\
& & $\operatorname{det}=\operatorname{Re}(B \bar{C})+O(u)$ \\
\end{tabular}

\section{THE TORUS BIFURCATION THEOREM}

In this section we prove the existence, direction of branching and asymptotic stability of certain invariant 2 -tori in codimension two bifurcations occurring in a class of symmetric systems of ODE. The general Theorems 4.5 and 4.6 are complemented by explicit formulas for computation that are derived in Section 5. A number of hypotheses are needed to prove our theorem and we describe them now. These hypotheses abstract properties of the $D_{n}$-equivariant systems described in Section 3. We return to $D_{3}$ symmetry in Sections 5 and 6 , where the results of this section are applied.

Specifically, we consider the two-parameter system of ODE

$$
\frac{d z}{d t}=f(z, \lambda, \mu)
$$

where $f: \mathbb{C}^{n} \times \mathbb{R}^{2} \rightarrow \mathbb{C}^{n}$ is smooth. The roles of the parameters $\lambda$ and $\mu$ are distinguished as follows. We assume that (4.1) undergoes a Hopf bifurcation as the primary bifurcation parameter $\lambda$ is varied and that a secondary torus bifurcates off of a primary branch of periodic solutions as $\lambda$ is further varied. The role of the auxiliary parameter $\mu$ is to allow the secondary torus bifurcation to coalesce with the primary Hopf bifurcation as $\mu$ is varied. We study here the simplest instances of such a codimension two bifurcation consistent with symmetry. We now state the hypotheses needed to define this simplest setting.

Our intention is to make a preliminary and naive discussion of degenerate Hopf bifurcations in the presence of symmetry. We do not pretend to have a general theory. A general theory, however, will have to include the examples and setting we study here. 


\subsection{Hypotheses on the Primary Hopf Bifurcation}

The simplest form of Hopf bifurcation in the presence of symmetry occurs under the following hypotheses (see Golubitsky and Stewart, 1985). We let $\Gamma$ be a finite subgroup of $O(n)$ and let $\Gamma$ act on $\mathbb{C}^{n}=\mathbb{R}^{n} \oplus i \mathbb{R}^{n}$ by $\gamma(x+i y)=\gamma x+i \gamma y$. The reason for restricting $\Gamma$ to be finite is discussed in $(\mathrm{H} 2)$ below. We assume that

$$
\Gamma \text { acts absolutely irreducibly on } \mathbb{R}^{n}
$$

that is, the only $n \times n$ real matrices commuting with $\Gamma$ are scalar multiples of the identity.

We assume that the $f$ in (4.1) commutes with $\Gamma$, undergoes a Hopf bifurcation at $\lambda=0$ when $\mu=0$, and is in Birkhoff normal form. The first and third of these assumptions are summarized by

$$
f \text { is } \Gamma \times S^{1} \text {-equivariant }
$$

where the circle group $S^{1}$ is viewed as the complex numbers of modulus one acting on $\mathbb{C}^{n}$ by complex multiplication. Thus

$$
f(\sigma z, \lambda, \mu)=\sigma f(z, \lambda, \mu) \quad \text { for all } \sigma \in \Gamma \times S^{1}
$$

Hypotheses (H1) and (H2) imply

$$
\begin{aligned}
f(0, \lambda, \mu) & =0 \\
f(-z, \lambda, \mu) & =-f(z, \lambda, \mu) \\
(d f)_{0, \lambda_{,} \mu} v & =A(\lambda, \mu) v \quad \text { for all } \quad v \in \mathbb{C}^{n}
\end{aligned}
$$

where $A(\lambda, \mu) \in \mathbb{C}$. Hypothesis (H1) implies that $\Gamma \times S^{1}$ acts irreducibly on $\mathbb{C}^{n}$ and hence (4.2a) is valid. Since $\pi \in S^{1}$ acts as -1 on $\mathbb{C}^{n}$, (H2) implies (4.2b). (H2) also implies that $(d f)_{0, \lambda, \mu}$ commutes with $\Gamma \times S^{1}$, from which (4.2c) follows.

The assumption that (4.1) undergoes a Hopf bifurcation at $\lambda=\mu=0$ implies that $A(0,0)$ is purely imaginary. We assume that periodic solutions to (4.1) are generated from this Hopf bifurcation is as simple a way as possible. We now describe this process. Begin by assuming that the eigenvalue $A(\lambda, 0)$ crosses the imaginary axis with nonzero speed, that is,

$$
\Delta_{3} \equiv \frac{\partial}{\partial \lambda}(\operatorname{Re} A)(0,0) \neq 0
$$

Assumption (H3) implies that for each $\mu$ near 0 there is a unique value $\lambda(\mu)$ 
at which $A(\lambda(\mu), \mu)$ is purely imaginary. For simplicity, and without loss of generality, we assume that $\lambda(u) \equiv 0$ so that

$$
A(0, \mu)=i \omega_{0}(\mu)
$$

where $\omega_{0}(0) \neq 0$. Thus, we assume that for each $\mu$ a Hopf bifurcation from the trivial steady state occurs in (4.1) at $\lambda=0$.

Let $\Sigma \subset \Gamma \times S^{1}$ be an isotropy subgroup. Golubitsky and Stewart (1985) show that a unique branch of periodic solutions to (4.1) with symmetry group $\Sigma$ can be found when

$$
\operatorname{dim} \operatorname{Fix}(\Sigma)=2
$$

Due to the assumption of Birkhoff normal form (H2), these periodic solutions all have the form

$$
z(t)=\varepsilon e^{i \omega t} p
$$

where $\varepsilon>0$ and

$$
\rho \in \operatorname{Fix}(\Sigma) \text { is chosen with }|p|=1
$$

Moreover, these periodic solutions are found by solving the equation in $\operatorname{Fix}(\Sigma)$

$$
f(\varepsilon p, \lambda, \mu)-i \varepsilon \omega p=0
$$

and assuming (H3), (4.5) can be solved uniquely for

$$
\begin{aligned}
\omega & =\omega^{*}\left(\varepsilon^{2}, \mu\right) \equiv \omega_{0}(\mu)+\omega_{2}(\mu) \varepsilon^{2}+\omega_{4}(\mu) \varepsilon^{4}+O\left(\varepsilon^{6}\right) \\
\lambda & =\lambda^{*}\left(\varepsilon^{2}, \mu\right)=\lambda_{2}(\mu) \varepsilon^{2}+\lambda_{4}(\mu) \varepsilon^{4}+O\left(\varepsilon^{6}\right)
\end{aligned}
$$

It follows from (4.6b) that this branch of periodic solutions is supercritical (in $\lambda$ ) if $\lambda_{2}(0)>0$ and subcritical if $\lambda_{2}(0)<0$. We assume

$$
\nabla_{6} \equiv \frac{1}{6}\left\langle\left(\partial^{3} f\right)_{0,0,0}(p, p, p), p^{*}\right\rangle \neq 0
$$

where (due to Birkhoff normal form) $p^{*}=p$ is an eigenvector of $(d f)_{0,0,0}^{*}$ with eigenvalue $\omega_{0}(0) i$. A calculation shows that

$$
\lambda_{2}(0)=-\Delta_{6} / \Delta_{3}
$$

To verify (4.7), substitute (4.6) in (4.5), set the coefficient of $\varepsilon^{3}$ in (4.6) to zero, and take the real part of the inner product with $p^{*}$. 


\subsection{Hypotheses on the Secondary Torus Bifurcation}

The assumption of Birkhoff normal form allows us to reduce the problem of finding periodic solutions of (4.1) to finding stationary solutions of (4.5). With this assumption the problem of finding a secondary torus bifurcation of (4.1) is reduced to finding a secondary Hopf bifurcation of (4.5). We now discuss the group theoretic restrictions on the action of $\Gamma$ which will admit the possibility of purely imaginary eigenvalues occurring in the linearization of (4.5) along the nontrivial branch of stationary solutions parametrized by (4.6).

Define

$$
g(z, \lambda, \mu, \omega)=f(z, \lambda, \mu)-i \omega z
$$

The linearization $d g$, evaluated at a solution (4.6), must commute with the isotropy subgroup $\Sigma \subset \Gamma \times S^{1}$. Let

$$
\mathbb{C}^{n}=V_{1} \oplus V_{2} \oplus \cdots \oplus V_{k}
$$

be the isotypic decomposition under $\Sigma$, that is, each of the $V_{j}^{\prime}$ 's is a sum of isomorphic irreducibile representations under $\Sigma$ and the irreducible representations of $\Sigma$ appearing in distinct $V$, s are themselves distinct. Since $\operatorname{Fix}(\Sigma)$ is the sum of all the trivial representations of $\Sigma$, we may take

$$
V_{1}=\operatorname{Fix}(\Sigma)
$$

Suppose now that $d g$ has a complex conjugate pair of purely imaginary eigenvalues. Generically, we expect the (generalized) eigenspace associated with these eigenvalues to be in some $V_{j}$; without loss of generality we can take $j=2$. The simplest type of torus bifurcation occurs when the purely imaginary eigenvalues are simple and the simplest way to force this hypothesis to be valid is to assume

$$
\operatorname{dim} V_{2}=2
$$

Let $L=d g \mid V_{2}$ restricted to the branch of solutions parametrized by (4.6). So

$$
L=L\left(\varepsilon^{2}, \mu\right)
$$

We comment on (4.11). Along the branch (4.6)

$$
z=\varepsilon p, \quad \lambda=\lambda^{*}\left(\varepsilon^{2}, \mu\right), \quad \text { and } \quad \omega=\omega^{*}\left(\varepsilon^{2}, \mu\right)
$$

Moreover, by (4.2b) and (4.8) $g$ is odd in $z$. Thus $d g$ is even in $z$ and hence in $\varepsilon$ and the form of $L$ given in (4.11) is valid. 
By (H7) $L$ is a linear mapping on a two-dimensional space. So $L$ has purely imaginary eigenvalues precisely when

$$
\operatorname{tr}(L)=0 \quad \text { and } \quad \operatorname{det}(L)>0
$$

We want to guarantee that for some $\mu$ near 0 , there are values of $\varepsilon^{2}$ for which $L$ has purely imaginary eigenvalues. That is, we want to guarantee the existence of solutions to the equation $\operatorname{tr} L\left(\varepsilon^{2}, \mu\right)=0$. Now observe that

$$
(d g)_{0,0, \mu, 0}=(d f)_{0,0, \mu}-i \omega_{0}(\mu) I=0
$$

by (4.2c) and (H4). Thus $L(0, \mu) \equiv 0$ and Taylor's theorem allows us to write

$$
L\left(\varepsilon^{2}, \mu\right)=\varepsilon^{2} L\left(\varepsilon^{2}, \mu\right)
$$

In order to guarantee the existence of solutions to $\operatorname{tr}(L)=0$, we assume

$$
\begin{aligned}
\operatorname{tr} L(0,0) & =0 \\
\Delta_{9} & \equiv \frac{\partial}{\partial \varepsilon^{2}}(\operatorname{tr} L)(0,0) \neq 0
\end{aligned}
$$

Hypotheses (H8) and (H9) along with the implicit function theorem guarantee a unique solution to

$$
\operatorname{tr} \mathrm{L}\left(\varepsilon^{2}, \mu\right)=0
$$

given by

$$
\varepsilon^{2}=E(\mu)
$$

Now solutions to (4.13) can exist only when $E(\mu) \geqslant 0$. Thus, to ensure the existence of such solutions, we assume

$$
\Delta_{10} \equiv \frac{\partial}{\partial \mu}(\operatorname{tr} \mathrm{L})(0,0) \neq 0
$$

Implicit differentiation of (4.13) shows that

$$
\frac{d E}{d \mu}(0)=-\Delta_{10} / \Delta_{9}
$$

Thus, a unique solution to (4.13) exists when $\mu$ is near 0 and

$$
\operatorname{sgn}(\mu)=-\operatorname{sgn}\left(\Delta_{9}\right) \operatorname{sgn}\left(\Delta_{10}\right)
$$


Finally, in order for $L$ to have purely imaginary eigenvalues, we must assume

$$
\operatorname{det} \mathrm{L}(E(\mu), \mu)>0
$$

for all $\mu$ near 0 satisfying (4.16). Hypothesis (H11) is the subject of further discussion below.

These 11 hypotheses describe the simplest situation where a secondary torus bifurcation of the type discussed at the beginning of this section might exist. In particular, these hypotheses guarantee the existence of a Hopf bifurcation along a nontrivial branch of the steady-state equation (4.5). However, as we now discuss, the existence of $\Gamma \times S^{1}$-symmetry in (4.1) ensures that the standard Hopf bifurcation theorem does not apply since the $S^{1}$-symmetry of normal form forces one eigenvalue of $d g \mid \operatorname{Fix}(\Sigma)$ to be zero. Observe that the group theoretic argument which guarantees that $d g$ has one zero eigenvalue also implies that $d g$ has $\operatorname{dim} \Gamma+1-\operatorname{dim} \Sigma$ zero eigenvalues.

Next we make the observation that group-theoretically there are two types of torus bifurcation. Consider the action of $\Sigma$ on $V_{2}$. The assumption that $L=d g \mid V_{2}$ can have purely imaginary eigenvalues, coupled with the fact that $L$ commutes with $\Sigma$, places restrictions on the action of $\Sigma$.

Let $K(\Sigma)$ be the kernel of the action of $\Sigma$ on $V_{2}$ and let $T(\Sigma)=$ $\Sigma / K(\Sigma)$. As observed by Golubitsky and Stewart (1985) either

(a) $V_{2}=\mathbb{R} \oplus \mathbb{R}$, where $T(\Sigma)$ acts absolutely irreducibly on $\mathbb{R}$, or

(b) $T(\Sigma)$ acts irreducibly, but not absolutely irreducibly, on $V_{2}$.

Moreover, since $V_{2} \cap \operatorname{Fix}(\Sigma)=\{0\}$ we know that $T(\Sigma)$ acts nontrivially on $V_{2}$. Hence in case (b), $T(\Sigma) \cong \mathbb{Z}_{q}$ for some $q \geqslant 3$, since these are the only finite groups which act faithfully, irreducibly, and not absolutely irreducibly on $\mathbb{R}^{2}$, and in case (a) $T(\Sigma) \cong \mathbb{Z}_{2}$.

Definition 4.1. When $T(\Sigma) \cong \mathbb{Z}_{2}$ we call the tori resulting from the secondary torus bifurcation standing tori and when $T(\Sigma) \cong \mathbb{Z}_{q}(q \geqslant 3)$ we call these rotating tori.

Observe that (H11) simplifies in the rotating tori case. There $\mathrm{L}\left(\varepsilon^{2}, \mu\right)$ commutes with $\mathbb{Z}_{q}$ and is hence a multiple of a rotation. Thus group theoretic restrictions force $\operatorname{det} \mathrm{L}\left(\varepsilon^{2}, \mu\right) \geqslant 0$ and (H11) simplifies to

$$
\Delta_{11}^{\mathrm{r}} \equiv \mathrm{L}(0,0) \neq 0
$$

The situation for standing tori is more complicated, as group restrictions may force $\mathrm{L}(0, \mu)=0$. In this case we find in examples that

$$
L\left(\varepsilon^{2}, \mu\right)=\varepsilon^{2 m} D\left(\varepsilon^{2}, \mu\right)
$$


where $m$ depends on $\Gamma$ and $\Sigma$ but not, in general, on the particular $f$ in (4.1). For standing tori we replace (H11) with

$$
\Delta_{11}^{\mathrm{s}} \equiv \operatorname{det} D(0,0)>0
$$

Note that (4.17) is valid for rotating waves; there $D=\mathrm{L}$ and $m=1$.

We end this section by explicitly constructing the action of $\Sigma$ on $V_{2}$. Observe that each $V_{j}$ is invariant under $S^{1}$, since $S^{1}$ commutes with the full group $\Gamma \times S^{1}$. Since $\operatorname{dim} V_{2}=2$ by (H7), it follows that we may identify $V_{2}$ with $\mathbb{C}$ and the action of $\Sigma$ on $V_{2}$ with a subgroup of $S^{1}$ acting on $\mathbb{C}$. Thus, the action of $T(\Sigma)$ on $\mathbb{C}$ is generated by

$$
z \rightarrow e^{2 \pi i / q} z
$$

for $q$ as defined above. (Note that standing tori correspond to $q=2$.)

\subsection{Hopf Bifurcation with Zero Eigenvalues}

In this section and Section 4.4 we prove a torus bifurcation theorem for vector fields satisfying (H1)-(H11). This theorem consists of two parts. In the first we show that the extra eigenvalue forced to be zero by symmetry in Hopf bifurcation causes no problems. Here we use results of Krupa (1988). In the second part we show how the direction of branching and the stability of the torus branch is determined by Taylor coefficients of $f$ at the origin. The computations in this section are example specific. We note that both of these parts could have been done in one step, as shown by Iooss (1983). We prefer the more geometrical exposition given here which separates theory and calculation. Recall that $f$ is assumed to be $\Gamma \times S^{1}$-equivariant and to have a periodic solution $z(t)=\varepsilon e^{i \omega t} p$.

We now concentrate on determining the form of $f$ on a neighborhood of the group orbit $X=\left(\Gamma \times S^{1}\right) \cdot p$. The existence of the periodic solution implies that $f$ is tangent to $X$ along $X$. We utilize two results from Krupa (1988). Let $N(X)$ denote the $\Gamma \times S^{1}$-equivariant normal bundle of $X \subset \mathbb{C}^{n}$, let $N_{x}$ denote the fiber over $x$, and let $\pi: N(X) \rightarrow X$ be the projection.

Theorem 4.2. There exist $\Gamma \times S^{1}$-equivariant vector fields $f_{\mathrm{T}}$ and $f_{N}$ such that

$$
f=f_{\mathrm{T}}+f_{N}
$$

where $f_{\mathrm{T}}(y)$ is in the tangent space to the group orbit of $\Gamma \times S^{1}$ through $y$ and $f(y) \in N_{\pi(y)}$. 
Theorem 4.3. Let $x(t)$ be the trajectory in $\dot{x}=f(x)$ with $x(0)=y$ and let $z(t)$ be the trajectory in $\dot{z}=f_{N}(z)$ with $z(0)=y$. Then

$$
x(t)=\delta(t) z(t)
$$

for some smooth curve $\delta(t) \in \Gamma \times S^{1}$ with $\delta(0)=$ identity.

It follows from Theorems 4.2 and 4.3 that a Hopf bifurcation to a periodic trajectory $z(t)$ for $f_{N}$ leads to a trajectory for $f$ on the union of group orbits through $z(t)$.

Remarks 4.4. (a) When $\Gamma$ is finite, all group orbits are circles and the flow is on 2-torus. The $S^{1}$-action forces the flow to be conjugate to linear. To lowest order, this flow has the form $\varepsilon e^{i \omega t} z(t)$. In perturbation theory language the flow will have the form

$$
\varepsilon e^{i(\omega+\theta) t}(p+h(t))
$$

where $\theta \in \mathbb{R}$ and $h(t) \in N_{p}$ are small. Moreover, $h(t)$ is a solution of

$$
\frac{d h}{d t}(t)=f_{N}(p+h(t), \lambda, \mu)
$$

To verify (4.21) let (4.20) be a solution to (4.1) and use the decomposition (4.18).

(b) Observe that

$$
g\left|N_{p}=f\right| N_{p}
$$

where $g$ is defined in (4.8). This follows since

$$
g=f-i \omega z=f_{N}+f_{\mathrm{T}}-i \omega z
$$

and $f_{\mathrm{T}}$ and $i \omega z$ vanish when restricted to $N_{p}$.

Theorem 4.5. Assume (H1)-(HI1). Then for fixed $\mu$ satisfying (4.15), there is a branch of periodic solutions to (4.1) parametrized by $\lambda$ which undergoes a torus bifurcation at $\lambda=\lambda^{*}$ as in (4.6b). When $\Gamma$ is finite, a unique branch of two-frequency trajectories bifurcate from the branch of periodic solutions at $\lambda=\lambda^{*}$.

Proof. Under our hypotheses the complex conjugate pair of eigenvalues of $L$ that cross through 0 as $\lambda$ is varied do so with nonzero speed. In fact, when $\mu$ is fixed,

$$
\frac{\partial}{\partial \lambda}(\operatorname{tr} L)=\frac{\partial}{\partial \varepsilon^{2}}(\operatorname{tr} L) \frac{\partial \varepsilon^{2}}{\partial \lambda}
$$


Both of these factors are nonzero, the first by (H9) and the second by (H3), (H6), and (4.7). It remains only to show that under the hypotheses above, the normal vector field $G=f_{N} \mid N_{p}$ undergoes a Hopf bifurcation at $\lambda=\lambda^{*}$. However, it is easy to show that the eigenvalues of $d G$ at $\varepsilon p$ are just the Floquet exponents of $f$ at the periodic solution $c e^{i \omega t} p$ minus $i \omega$. Thus the eigenvalues of $d G$ at $\varepsilon p$ are the eigenvalues of $d g$ in the direction $N_{p}$ [where $g$ is defined in (4.8)]. Hence our hypotheses imply that $f_{N}$ undergoes a (simple) Hopf bifurcation at $\lambda=\lambda^{*}$ and that the corresponding complex eigenvalues cross the imaginary axis with nonzero speed. The standard Hopf theorem coupled with Remark (4.4a) now applies.

\subsection{Direction and Stability of the Branch of Invariant 2-Tori}

Next we consider the direction of branching of the branch of 2-tori by determining the direction of branching of the branch of periodic solutions in the Hopf bifurcation of $G=f_{N} \mid N_{p}$. Theorem 4.3 implies that asymptotic stability of the periodic solutions of $G$ in $N_{p}$ implies asymptotic stability of the invariant 2 -tori in (4.1).

We review the relevant discussion from the previous sections. Let $y=$ $\varepsilon(p+h)$. There is a branch of equilibria of $f_{N}$ at

$$
h=0 \quad \text { and } \quad \lambda=\lambda^{*}\left(\varepsilon^{2}, \mu\right)
$$

where $\mu^{*}$ is defined by (4.16). A Hopf bifurcation for $f_{N}$ occurs along this branch of equilibria at

$$
\varepsilon^{2}=E(\mu)
$$

as defined in (4.14), since $V_{2} \subset N_{p}$. Note that $E(0)=0$. In fact, $\mathrm{L}\left(\varepsilon^{2}, \mu\right)$ is just $d f_{N} \mid V_{2}$.

We assume that $\mu$ has the correct sign so that $(4.16)$ is valid, and hence (4.25) has a solution for $\varepsilon^{2}$ when $E(\mu)$ is positive. We have assumed, moreover, that as $\lambda$ varies through $\lambda^{*}$, the complex eigenvalues of $\mathrm{L}$ cross the imaginary axis with nonzero speed, as noted in the proof of Theorem 4.5.

The standard Hopf theorem asserts that there exists a single coefficient $\mu_{2}$, depending on terms of $f_{N}$ through cubic order, that determines the asymptotic stability of the periodic solutions (and their direction of branching). Moreover, $\mu_{2}$ is defined at the point of Hopf bifurcation given by (4.24) and (4.25). Thus

$$
\mu_{2}=\mu_{2}(\mu)
$$


Theorem 4.6. Under the assumptions just described

$$
\mu_{2}(\mu)=\mu^{k} M(\mu)
$$

for some integer $k$. A formula for $M(0)$ can be determined, in principal, from the Taylor expansion of $f$ at the origin and the sign of $M(0)$ determines the asymptotic stability and direction of branching of the branch of 2-tori for (4.1). In particular, this branch is supercritical and consists of asymptotically stable 2-tori when $M(0)>0$ and the branch is subcritical when $M(0)<0$.

Our final (genericity) assumption is

$$
M(0) \neq 0
$$

The most difficult part of any calculation of invariant 2-tori is determining $M(0)$, that is, determining the direction of branching and stability of the 2-tori. In principle, it might be possible to derive a general formula for $M(0)$ when $k=1$ using only terms in the Taylor expansion of $f$. In our $D_{3}$ example in the next section we have chosen the computationally simpler route of just computing the secondary Hopf bifurcation on $f_{N}$ directly. One reason is that we find that $k=1$ is valid for the standing tori and $k=2$ is valid for the rotating tori. At this stage we do not understand why certain isotropy subgroups force $k$ to be greater than 1 .

\section{TORUS BIFURCATIONS WITH TRIANGULAR SYMMETRY}

In this section we apply our torus bifurcation theorem to a vector field

$$
\frac{d z}{d t}=g(z, \lambda, \mu)
$$

in $D_{n} \times S^{1}$-normal form, that is, we assume $g$ has the form (3.6). As we discussed in Section 3 (see Table I), generically there exist three primary branches of periodic solutions to (5.1) corresponding to two-dimensional fixed point subspaces. We need to determine when hypotheses $(\mathrm{H} 1-\mathrm{H} 12)$ are valid for each of these branches. Note that hypotheses (H1)-(H3), $(\mathrm{H}(5)$, and $(\mathrm{H} 7)$ are automatically valid in these cases.

In (3.6) the invariant functions $A-D$ are complex-valued and we denote these functions by

$$
\begin{aligned}
& A=a+i \alpha \\
& B=b+i \beta \\
& C=c+i \gamma \\
& D=d+i \delta
\end{aligned}
$$


Hypothesis (H4) states that a Hopf bifurcation occurs at $\lambda=\mu=0$ and that the complex eigenvalue crosses the axis with nonzero speed. We simplify our analysis here by assuming

$$
a=\lambda+\{\text { a function depending on } z \text { alone }\}
$$

and that $\alpha(0) \equiv \alpha_{0}>0$. Thus, we assume that the trivial steady state $z=0$ is asymptotically stable when $\lambda<0$ and loses stability at $\lambda=0$. Moreover, (H4) is valid as $\Delta_{3}=1 \neq 0$.

In our analysis, we begin by assuming that $n \neq 4$. The case of square symmetry $(n=4)$ is more complicated (see Golubitsky and Stewart, 1986; Swift, 1988). In particular, when $n \neq 4$ the standing waves are either both supercritical or both subcritical, and there are no branches of periodic solutions corresponding to submaximal isotropy. The criticality of these branches is determined by $\operatorname{sgn}\left(\Delta_{6}\right)$, as noted in Table III. Assuming $b \neq-a_{N},-2 a_{N}$ validates (H6).

The degeneracy condition needed to have a torus bifurcation, hypothesis ( $\mathrm{H} 8$ ), is $\operatorname{tr} \mathrm{L}=0$. For each of the branches, (H8) corresponds to $b=0$ at the origin. This could have been seen directly from the stability results of Golubitsky and Stewart (1986) since the coefficient $b(0)$ being nonzero was needed to determine which of these branches of periodic solution could be asymptotically stable. Assuming $b(0)=0$ implies that standing waves and rotating waves must be all supercritical or subcritical, depending on the sign of $a_{N}(0)$, which is assumed to be nonzero.

We again simplify our analysis by assuming that

$$
b(z, \mu)=\mu+\{a \text { function depending only on } z\}
$$

It then follows that $\Delta_{10}=-1$ for rotating waves and +1 for standing waves. Thus $(\mathrm{H} 10)$ is valid and we will have a torus bifurcation if the complex eigenvalues in the $V_{2}$-directions are nonzero since $(\mathrm{H} 10)$ implies that these eigenvalues will cross the imaginary axis with nonzero speed. That these eigenvalues will be nonzero and purely imaginary at the point of secondary bifurcation is governed by (H11). Thus we assume $\beta(0) \gamma(0) \neq 0$, as indicated in Table III.

In our discussion in Section 4 we also assumed that we could solve uniquely for the point of the secondary bifurcation, as a function of $\mu$, which follows from $A_{9} \neq 0$. So we assume $b_{N}(0) \neq 0, \pm 2 c(0)$, and (H9) is verified. [Note that when $n \geqslant 5$, this condition would be $b_{N}(0) \neq 0$.]

We summarize our discussion by listing all conditions in Table IV.

The final issue we must address is the direction of branching of the secondary branch of 2-tori, hypothesis (H12). At the end of Section 4, we discussed the difficulty of deriving a formula for the direction of branching 
Table III. Data Needed to Find Torus Bifurcations Along Primary Branches in Degenerate $D_{3} \times S^{1}$-Equivariant Hopf Bifurcation (All Functions Are Evaluated at the Origin)

\begin{tabular}{cccc}
\hline$\Sigma$ & Rotating wave & Standing wave 1 & Standing wave 2. \\
\hline $\operatorname{Fix}(\Sigma)$ & $\left(z_{1}, 0\right)$ & $\left(z_{1}, z_{1}\right)$ & $\left(z_{1},-z_{1}\right)$ \\
$\operatorname{sgn}\left(\Delta_{6}\right)$ & $-\left(a_{N}+b\right)$ & $-\left(2 a_{N}+b\right)$ & $-\left(2 a_{N}+b\right)$ \\
$V_{2}$ & $\left(0, z_{2}\right)$ & $\left(z_{2},-z_{2}\right)$ & $\left(z_{2}, z_{2}\right)$ \\
$\operatorname{tr} \mathrm{L}=0$ & $b=0$ & $b=0$ & $b=0$ \\
$\operatorname{sgn}\left(\Delta_{9}\right)$ & $-b_{N}$ & $b_{N}-2 c$ & $b_{N}+2 c$ \\
$\Delta_{10}$ & $-b_{\mu}$ & $b_{\mu}$ & $b_{\mu}$ \\
$(\mathrm{H} 11)$ & $\beta \neq 0$ & $\beta \gamma<0$ & $\beta \gamma>0$ \\
\hline
\end{tabular}

of the torus branches. Because of this fact, we compute, in Sections 5.1 and 5.2, the direction of branching of the secondary 2-tori bifurcation along the rotating and standing waves branches only in the case of $D_{3}$-symmetry. Let $\beta_{0} \equiv \beta(0)$ and $\gamma_{0} \equiv \gamma(0)$. We prove the following.

Theorem 5.1. In degenerate $D_{3}$-equivariant Hopf bifurcation, the direction of branching of the branch of rotating 2-tori is supercritical if

$$
-2 a_{N}\left[b_{N}+\frac{\gamma_{0}}{3 \beta_{0}}\right]
$$

is positive and subcritical if it is negative.

Theorem 5.2. In degenerate $D_{3}$-equivariant Hopf bifurcation, the direction of branching of the branch of standing 2-tori off of the branch of periodic standing waves 1 is supercritical if

$$
-\frac{\beta_{0}}{3 \gamma_{0}}\left[\operatorname{sgn}\left(\gamma_{0}\right) \frac{\beta_{0}^{2}}{2 a_{N} \sqrt{6\left|\beta_{0} \gamma_{0}\right|}}+\frac{4 a_{N}}{3}\right] \frac{a_{N}}{2 c_{0}-b_{N}}
$$

is positive and subcritical if it is negative. For secondary bifurcation off of standing waves 2 we replace (5.6) by

$$
-\frac{\beta_{0}}{3 \gamma_{0}}\left[\operatorname{sgn}\left(\gamma_{0}\right) \frac{\beta_{0}^{2}}{2 a_{N} \sqrt{6\left|\beta_{0} \gamma_{0}\right|}}-\frac{4 a_{N}}{3}\right] \frac{a_{N}}{2 c_{0}-b_{N}}
$$

It is possible to derive the direction of branching of standing torus 2 from that of standing torus 1 usinfg the following observation first noted by Swift (1988), using the terminology "parameter" symmetry.

We call the mapping

$$
Q\left(z_{1}, z_{2}\right)=\left(z_{1},-z_{2}\right)
$$


Table IV. Conditions for Torus Bifurcation with $D_{3}$-Symmetry
(a) $a(0)=0, \alpha(0)>0$
Primary Hopf bifurcation
(b) $a_{N}(0)>0$
Periodic solutions subcritical
(c) $b(0)=0$
Possibility of secondary torus bifurcation
(d) $\beta(0) \neq 0$
Torus bifurcation on rotating waves
(e) $\beta(0) \gamma(0)<0(>0)$
Torus bifurcation on standing wave 1
(standing wave 2)
(f) $b_{N}(0) \neq 0, \pm 2 c(0)$
See (4.16): unique torus bifurcation along rotating wave when $\operatorname{sgn}(\mu)=-\operatorname{sgn}\left(b_{N}(0)\right)$
standing wave 1 when $\operatorname{sgn}(\mu)=-\operatorname{sgn}\left(b_{N}(0)-2 c(0)\right)$
standing wave 2 when $\operatorname{sgn}(\mu)=-\operatorname{sgn}\left(b_{N}(0)+2 c(0)\right)$

a quasisymmetry since it is in the normalizer of $\Gamma$ in $O(4)$ but is not in $\Gamma=D_{3} \times S^{1}$. It follows that the map

$$
h=Q g(Q z)
$$

is $\Gamma$-equivariant whenever $g$ is $\Gamma$-equivariant. In this particular case, the quasisymmetry $Q$ interchanges standing waves 1 and 2 . Since $h$ is vector field equivalent to $g$, the dynamics of $h$ is the same as that of $g$. Thus, computing the direction of branching of standing torus 1 for $h$ gives the direction of branching of standing torus 2 for $g$.

It remains only to note using (3.4) and (3.6) that when $n=3, g$ transforms to $h$ in (5.9) as follows:

$$
\begin{aligned}
& (N, P, S, T) \rightarrow(N, P,-S,-T) \\
& (A, B, C, D) \rightarrow(A, B,-C,-D)
\end{aligned}
$$

Thus (5.7) may be derived from (5.6) by transforming $\gamma_{0}$ to $-\gamma_{0}$.

\subsection{Rotating Tori for $D_{3}$-Symmetry}

We let $\Sigma$ be the isotropy subgroup of rotating waves

$$
\left.\tilde{\mathbb{Z}}_{3}=\{(\gamma,-\gamma)\}: \gamma \in \mathbb{Z}_{3}\right\}
$$

with the two-dimensional fixed point space $\left\{\left(z_{1}, 0\right)\right\}$. The branching equation for the periodic solutions lying in $\operatorname{Fix}(\Sigma)$ is given by

$$
A+B u^{2}=0, \quad u \in \mathbb{R}
$$


From our discussion above concerning the torus bifurcation, we may assume that the system of ODE (5.1) has the form

$$
\begin{aligned}
\frac{d}{d t}\left[\begin{array}{l}
z_{1} \\
z_{2}
\end{array}\right]= & \left\{i \alpha_{0}+\lambda+a_{N} N+a_{N N} N^{2} / 2+a_{P} P\right\}\left[\begin{array}{l}
z_{1} \\
z_{2}
\end{array}\right] \\
& +\left\{\mu+b_{N} N+i \beta_{0}\right\}\left[\begin{array}{l}
z_{1}^{2} \bar{z}_{1} \\
z_{2}^{2} \bar{z}_{2}
\end{array}\right]+\left\{c_{0}+i \gamma_{0}\right\}\left[\begin{array}{l}
\bar{z}_{1}^{2} z_{2}^{3} \\
z_{1}^{3} \bar{z}_{2}^{2}
\end{array}\right]+\text { h.o.t. }
\end{aligned}
$$

where $N=\left|z_{1}\right|^{2}+\left|z_{2}\right|^{2}$ and $P=\left|z_{1}\right|^{2}\left|z_{2}\right|^{2}$. The branch of periodic solutions to (5.1) within $\operatorname{Fix}\left(\widetilde{\mathbb{Z}}_{3}\right)$ is given by:

$$
z_{\varepsilon}(t)=\varepsilon\left[\begin{array}{l}
1 \\
0
\end{array}\right] e^{i \omega^{*} t}
$$

where $\omega=\omega^{*}\left(\varepsilon^{2}, \mu\right)=\alpha_{0}+\beta_{0} \varepsilon^{2}+O\left(\varepsilon^{4}\right)$. Along the branch of periodic solutions, the eigenvector due to translation, corresponding to the eigenvalue zero, is constant. In fact (5.12) shows that this eigenvector equals ip where $p=(1.0)^{T}$. We exploit this fact to reduce explicity the vector field to the normal section. We let

$$
V_{p}=\left\{z \in \mathbb{C}^{2}: \operatorname{Re}\langle z, i p\rangle=0\right\}
$$

By the implicit function theorem we can solve locally in (5.15a) for $\theta=\theta^{*}(h, \varepsilon, \mu)$ such that

$$
\begin{gathered}
\operatorname{Re}\left\langle f\left(\varepsilon(p+h), \lambda^{*}, \mu\right)-i\left(\omega^{*}+\theta\right) \varepsilon(p+h), i p\right\rangle=0 \\
\theta^{*}(0,0,0)=0
\end{gathered}
$$

If we now let

$$
g(h, \varepsilon, \mu, \theta)=f\left(\varepsilon(p+h), \lambda^{*}(\varepsilon, \mu), \mu\right)-i\left(\omega^{*}(\varepsilon, \mu)+\theta^{*}\right) \varepsilon(p+h)
$$

then the subspace $V_{P}$ is invariant under $g$ and $g \mid V_{P}$ is the normal component of the vector fieid $g$. For the details of this reduction see Vanderbauwhede et al. (1988) and Krupa (1988).

We note that $\theta^{*}$ corresponds to an element $\eta$ of the Lie algebra $L(\Gamma)$ and determines the drift along the group orbits.

In the language of asymptotic expansions we are looking for solutions of the form

$$
\varepsilon e^{i\left(\omega^{*}+e^{3} \theta\right) t}\left(p+h\left(\varepsilon^{2} \beta t\right)\right)
$$

where $h$ is a $2 \pi$-periodic function. 
In $\left(z_{1}, z_{2}\right)$ coordinates we write $h=\left(h_{1}, h_{2}\right)$ and $h_{1}=x+i y$. The normal vectorfield then is given by

$$
\begin{aligned}
\frac{d x}{d t}= & \varepsilon^{2}\left[2\left(a_{N}+\mu\right) x+a_{N}\left|h_{2}\right|^{2}+O\left[|x|^{2}+|x|\left|h_{2}\right|^{2}+\left|h_{2}\right|^{4}+\varepsilon^{2}|x|\right)\right] \\
\frac{d h_{2}}{d t}= & \varepsilon^{2}\left[-\left(\mu+i \beta_{0}+i \frac{\theta^{*}}{\varepsilon^{2}}\right) h_{2}+2 a_{N} x h_{2}+a_{N} h_{2}\left|h_{2}\right|^{2}\right. \\
& \left.+O\left(\varepsilon^{2}+|\mu|\left|h_{2}\right|^{3}+\left|h_{2}\right|^{3}\right)\right]
\end{aligned}
$$

We remark that $\theta^{*}$ is of order $\varepsilon^{2}$ and occurs in the second equation in such a way that its value will influence only the period of the bifurcating periodic solution, and not its stabililty. Therefore, we may suppress $\theta^{*}$.

More importantly, we observe that the direction of branching is determined by the higher-order terms. To see this, we rescale the time by letting $\vec{t}=-\varepsilon^{2} \beta_{0} t$ and eliminate the $\varepsilon^{2}$ which factors $L(\varepsilon, \mu)$ [see (4.11)]. We obtain

$$
\begin{aligned}
& -\beta_{0} \frac{d x}{d t}=2\left(a_{N}+\mu\right) x+a_{N}\left|h_{2}\right|^{2}+\text { h.o.t. } \\
& -\beta_{0} \frac{d h_{2}}{d t}=-\left(\mu+i \beta_{0}\right) h_{2}+\left(2 a_{N} x+\left(a_{N}+i \beta_{0}\right)\left|h_{2}\right|^{2}\right) h_{2}+\text { h.o.t. }
\end{aligned}
$$

From the first equation we see that $x=-\left|h_{2}\right|^{2} / 2+\ldots$, and inserting this in the second equation, we conclude that the direction of branching is not determined. To the next order in $\varepsilon^{2}$ we get (keeping the rescaled time)

$$
\begin{aligned}
-\beta_{0} \frac{d x}{d t}= & 2\left(a_{N}+\mu\right) x+a_{N}\left|h_{2}\right|^{2}+\varepsilon^{2}\left(2\left(a_{N N}+2 b_{N}\right) x+\left(a_{N N}+b_{N}\right)\left|h_{2}\right|^{2}\right) \\
& +O\left(\varepsilon^{2}\left(|x|+\left|h_{2}\right|^{2}\right)^{2}+\varepsilon^{4}\right) \\
-\beta_{0} \frac{d h_{2}}{d t}= & -\left(\mu+i \beta_{0}+\varepsilon^{2} b_{N}\right) h_{2}+\left(a_{N}+i \beta_{0}\right)\left|h_{2}\right|^{2} h_{2}+2 a_{N} x h_{2} \\
& +\varepsilon^{2}\left(2 a_{N N} x h_{2}+\left(c_{0}+i \gamma_{0}\right) \bar{h}_{2}^{2}+\left(a_{N N}+b_{N}\right)\left|h_{2}\right|^{2} h_{2}\right) \\
& +O\left(\varepsilon^{2}|\mu|\left|h_{2}\right|^{3}+\varepsilon^{2}\left(|x|+\left|h_{2}\right|^{2}\right)^{2}+\varepsilon^{4}\right)
\end{aligned}
$$

From (5.17) we derive that at a periodic solution

$$
x=\frac{-a_{N}+\varepsilon^{2} b_{N}}{2 a_{N}}\left|h_{2}\right|^{2}+O\left(\varepsilon^{2}\left|h_{2}\right|^{4}+\varepsilon^{4}\right)
$$


Then we find the direction of branching from (5.18)

$$
\mu=\varepsilon^{2}\left\{-b_{N}+2\left|h_{2}\right|^{2}\left[\frac{\gamma_{0}}{3 \beta_{0}}+b_{N}\right]+O\left(\varepsilon^{2}+\left|h_{2}\right|^{2}\right)\right\}
$$

From this equation Theorem 5.1 follows easily.

\subsection{Standing Tori for $D_{3}$-Symmetry}

We let $\Sigma$ be the isotropy subgroup $\mathbb{Z}_{2} \oplus \mathbb{Z}_{2}^{c}$ of standing waves 1 with two-dimensional fixed point space $\left\{\left(z_{1}, z_{1}\right)\right\}$. In order to construct $V_{P}$ and $\theta^{*}$ as in the rotating case, we first change coordinates in (5.12): $u=$ $\left(z_{1}+z_{2}\right) / \sqrt{2}, v=\left(z_{1}-z_{2}\right) / \sqrt{2}$.

The construction of the reduced vector field is mutatis mutandis the same as in the case of the rotating tori. Again, we suppress $\theta^{*}$, because we are interested only in the direction of branching. The effect of the change of coordinates is that the primary branch has the same form:

$$
z_{\varepsilon}(t)=\left[\begin{array}{l}
1 \\
0
\end{array}\right] e^{i \omega * t}
$$

where is the standing wave case

$$
\omega^{*}\left(\varepsilon^{2}, \mu\right)=\alpha_{0}+\frac{1}{2} \beta_{0} \varepsilon^{2}+O\left(\varepsilon^{4}\right)
$$

It occurs at

$$
\lambda^{*}\left(\varepsilon^{2}, \mu\right)=-\left(a_{N}+\frac{\mu}{2}\right) \varepsilon^{2}-\left(a_{N N}+\frac{a_{P}}{2}+b_{N}+\frac{c_{0}}{2}\right) \varepsilon^{4} / 2
$$

The reduced vector field has the form (compare with (5.12))

$$
\begin{aligned}
& \frac{d x}{d t}=\varepsilon^{2} f\left(x, h_{2}, \mu, \varepsilon^{2}\right) \\
& \frac{d h_{2}}{d t}=\varepsilon^{2} g\left(x, h_{2}, \mu, \varepsilon^{2}\right)
\end{aligned}
$$

The linear part of $g$ depends only on $h_{2}$. It is given by the mapping from $\mathbb{C}^{2}$ into itself by

$$
\begin{aligned}
v \rightarrow & {\left[\frac{\left(\mu+i \beta_{0}\right)}{2}+\left(\frac{b_{N}}{2}-c_{0}-i \gamma_{0}\right) \varepsilon^{2}\right] v } \\
& +\left[\frac{\left(\mu-i \beta_{0}\right)}{2}+\frac{\left(b_{N}-c_{0}+i \gamma_{0}\right)}{2} \varepsilon^{2}\right] \bar{v}+O\left(\varepsilon^{4}\right)
\end{aligned}
$$


If we consider this mapping as a mapping on $\mathbb{R}^{2}$, then its trace equals

$$
\mu+\left(b_{N}-2 c_{0}\right) \varepsilon^{2}+O\left(\varepsilon^{4}\right)
$$

and its determinant equals

$$
-\frac{3}{2}\left(\mu c_{0}-\gamma_{0} \beta_{0}\right) \varepsilon^{2}+O(\varepsilon)^{4}
$$

We apply an $\varepsilon$-dependent transformation to put the linear part of the vector field into normal form. Writing $h_{2}=u+i v$ the transformation is

$$
\begin{aligned}
& u \rightarrow \varepsilon u \\
& v \rightarrow \sqrt{-2 \beta_{0} / 3 \gamma_{0}} v
\end{aligned}
$$

We rescale the time by $\varepsilon$, i.e., $\varepsilon t=\bar{t}$. After these transformations the linear part of the vector field has the form

$$
\begin{aligned}
\frac{d x}{d t} & =\varepsilon\left(2 a_{N}+\mu\right) x \\
\frac{d h_{2}}{d t} & =\varepsilon \frac{\mu}{2}\left(h_{2}+\bar{h}_{2}\right)+i \varepsilon^{2} \tau_{0} h_{2}
\end{aligned}
$$

where $\tau_{0}=\sqrt{-3 \beta_{0} \gamma_{0} / 2}$. We rescale the time once more, $\varepsilon^{2} \tau_{0} t=\bar{t}$. Then the linear vector field has a circle of periodic solutions:

$$
\left[\begin{array}{l}
x \\
h_{2}
\end{array}\right]=\left[\begin{array}{c}
0 \\
\eta e^{i(s+\phi)}
\end{array}\right], \quad \phi \in S^{1} \cdot \eta \in \mathbb{R}^{+}
$$

We then put the full nonlinear vector field up to order three in $\left(x, h_{2}\right)$ into Birkhoff normal form. Phrased differently, after a near identity transformation in the variables $\left(x, h_{2}\right)$ and a truncation at order three, the resulting vector field has the form

$$
\begin{aligned}
& \varepsilon \frac{a x}{d t}=\tilde{f}\left(x,\left|h_{2}\right|^{2}, \mu, \varepsilon\right) \\
& \varepsilon^{2} \frac{d h_{2}}{d t}=\tilde{g}\left(x,\left|h_{2}\right|^{2}, \mu, \varepsilon\right) h_{2}
\end{aligned}
$$

If we can solve the equation $\widetilde{f}=\tilde{g}=0$, then we will know the direction of branching. Straightforward (but rather lengthy) computations show that

$$
\tilde{f}=2 a_{N} \xi+\frac{1}{3 \gamma_{0}} a_{N}\left|h_{2}\right|^{2} \beta_{0}+O\left[\left(\varepsilon^{2}+|\mu|\right)\left(|x|+\left|h_{2}\right|\right)+|x|\left|h_{2}\right|+\left|h_{2}\right|^{3}+|x|^{2}\right]
$$


where

$$
\xi=x+\frac{\varepsilon i}{6 \gamma}\left(h_{2}^{2}-h_{2}^{-2}\right) \beta_{0}
$$

Therefore, at a solution of $\tilde{f}=0$ we will have that

$$
x=-\frac{\beta_{0}}{6 \gamma_{0}}\left[\left|h_{2}\right|^{2}-\frac{\varepsilon i}{2 a_{N}}\left(h_{2}^{2}-h_{2}^{-2}\right)\right]+O\left(\varepsilon^{2}\left|h_{2}\right|+\left|h_{2}\right|^{3}\right)
$$

Substiting into the equations $\tilde{g}=0$ then yields

$$
\mu=\left(2 c_{0}-b_{N}\right) \varepsilon^{2}-\left|h_{2}\right|^{2}\left(\frac{4 \beta_{0} a_{N}}{3 \gamma_{0}}+\frac{\beta_{0}^{3}}{18 \kappa a_{N} \gamma_{0}^{2}}\right)+O\left(|\varepsilon|+\left|h_{2}\right|\right)^{3}
$$

where $\kappa=\left(-2 \beta_{0} / 3 \gamma_{0}\right)^{1 / 2}$. From this equation Theorem 5.2 follows.

The (above-mentioned) lengthy calculations were checked with the formula manipulation program REDUCE.

\section{THE BIFURCATION DIAGRAMS}

The results in Section 5 imply that when deriving the bifurcation diagrams for the torus bifurcation in degenerate Hopf bifurcation with $D_{3}$ symmetry, we may assume

$$
\begin{aligned}
& A=\hat{\lambda}+a_{N} N+\alpha_{0} i \\
& B=\mu+b_{N} N+\beta_{0} i \\
& C=c_{0}+\gamma_{0} i \\
& D=0
\end{aligned}
$$

as only these terms enter the determination of direction of branching and stability.

We assume

$$
\alpha_{0}>0, \quad \beta_{0}>0, \quad \gamma_{0}<0, \quad \text { and } \quad a_{N}<0
$$

We make these choices for the following reasons. First, without loss of generality, the frequencies $\alpha_{0}$ and $\beta_{0}$ may be assumed to be positive. Second, the quasisymmetry $Q$ transforms $\gamma_{0}$ to $-\gamma_{0}$ (and $c_{0}$ to $-c_{0}$ ), so we may assume that $\gamma_{0}$ is negative (at the expense of interchanging the two branches of standing waves). Finally, we are interested mainly in those situations where asymptotically stable states may exist. Indeed, stability can occur only when the primary branches are supercritical; hence we assume $a_{N}<0$. 
To simplify subsequent calculations rescale time and space to obtain

$$
\beta_{0}^{2}=1 / 2 \quad \text { and } \quad \gamma_{0}=-3 \beta_{0}
$$

We now find that when a torus bifurcation occurs, it occurs at

$$
\begin{array}{ll}
\lambda_{\mathrm{r}}=\frac{a_{N}}{b_{N}} \mu+\cdots & \text { (rotating torus) } \\
\lambda_{\mathrm{s}}=\frac{a_{N}}{b_{N}-2 c_{0}} \mu+\cdots & \text { (starting torus) }
\end{array}
$$

and the direction of branching is determined by

$$
\begin{aligned}
1-\frac{1}{b_{N}} & \text { (rotating torus) } \\
\frac{1}{b_{N}-2 c_{0}}\left[4 a_{N}-\frac{1}{4 a_{N}}\right] & \text { (standing torus) }
\end{aligned}
$$

where supercritical is positive and subcritical is negative.

We note that it is possible to choose coefficients independently so that, for a fixed $\mu$,

(i) standing wave 1 is stable at the initial bifurcation (choose $\mu<0$ ),

(ii) both a rotating and a standing torus bifurcate (choose $b_{N}>0$ and $b_{N}>2 c_{0}$ ),

(iii) either torus may bifurcate first as $\lambda$ increases (choose $c_{0}>0$ to have standing wave first, $c_{0}<0$ for rotating wave),

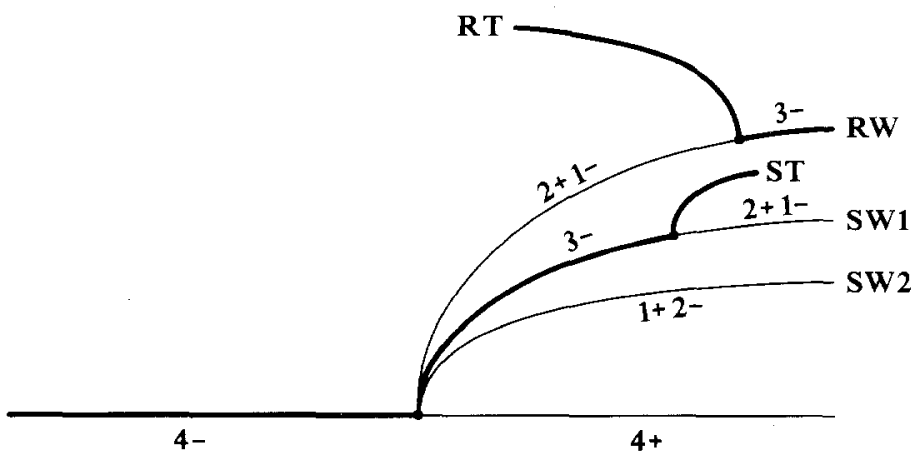

Fig. 1. Bifurcation diagram indicating values of $\lambda$ having two branches of asymptotically stable 2-tori and no stable steady states or periodic solutions. 
(iv) the rotating torus is either supercritical or subcritical (choose $b_{N}>1$ or $b_{N}<1$ ), and

(v) the standing torus is either supercritical or subcritical (choose $a_{N}<0.25$ or $0>a_{N}>-0.25$ ).

Therefore, it is possible to choose parameters so that the bifurcation diagram pictured in Fig. 1 occurs. Here we find the possibility of two stable 2-tori and no stable periodic solutions. Note that this phenomenon may not occur in codimension two for $D_{n}$-Hopf bifurcation when $n \geqslant 5$, since $c_{0}$ is then a higher-order term.

\section{ACKNOWLEDGMENTS}

This research was supported in part by the ACMP program of DARPA (MG), by NASA/Ames Grant NAG 2-432 (MG and SvG), and by NSF Grant DMS-8700897 (MG). The first author would also like to thank the University of Houston for a visiting position during which time some of the research reported here was completed.

\section{REFERENCES}

Chossat, P. (1986). Remarques sur la bifurcation secondaire de solutions quasi-périodiques dans un problème de bifurcation de Hopf de codimension 2 et invariant par symétrie O(2). C. R. Acad. Sci. Paris 302, 539-541.

Chossat, P., and Golubitsky, M. (1988). Iterates of maps with symmetry. SIAM J. Math. Anal. 19, No. 6, 1259-1270.

Chossat, P., and Iooss, G. (1985). Primary and secondary bifurcations in the Couette-Taylor problem. Jap. J. Appl. Math. 2, No. 1, 37-68.

Crawford, J. D., and Knobloch, E. (1988). Classification and unfolding of degenerate Hopf bifurcations with $O(2)$ symmetry: No distinguished parameter, Physica D 31, $1-48$.

Erneux, T., and Matkowsky, B. J. (1984). Quasi-periodic waves along a pulsating propagating front in a reaction-diffusion system. SIAM J. Appl. Math. 44, 536-544.

Golubitsky, M., and Guckenheimer, J. (1986). Multiparameter Bifurcation Theory, Contemporary Mathematics 56, Am. Math. Soc., Providence, R.I.

Golubitsky, M., and Langford, W. F. (1981). Classification and unfoldings of degenerate Hopf bifurcations. J. Diff. Eq. 41, 375-415.

Golubitsky, M., and Roberts, M. (1987). A classification of degenerate Hopf bifurcations with $O(2)$ symmetry. J. Diff. Eq. 69, No. 2, 216-264.

Golubitsky, M., and Stewart, I. N. (1985). Hopf bifurcation in the presence of symmetry. Arch. Rational Mech. Anal. 87, No. 2, 107-165.

Golubitsky, M., and Stewart, I. N. (1986). Hopf bifurcation with dihedral group symmetry: Coupled nonlinear oscillators. In Golubitsky, M., and Guckenheimer, J. (eds.), Multiparameter Bifucation Series, Contemporary Mathematics 46, Am. Math. Soc., Providence, R.I. pp. 131-173.

Golubitsky, M., Stewart, I., and Schaeffer, D. G. (1988). Singularities and Groups in Bifurcation Theory, Vol. II, Appl. Math. Sci. Ser. 69, Springer-Verlag, New York. 
Iooss, G. (1979). Bifurcation of Maps and Applications, Mathematical Studies 36, NorthHolland, Amsterdam.

Iooss, G. (1983). Bifurcation Theory and Applications, Lect. Notes Math. 1057, SpringerVerlag, New York.

Knobloch, E. (1986). On the degenerate Hopf bifurcation with $O(2)$ symmetry. In Golubitsky, M., and Guckenheimer, J. (eds.), Multiparameter Bifurcation Series, Contemporary Mathematics 56, Am. Math. Soc., Providence, R.I. pp. 193-202.

Krupa, M. (1988). Bifurcation from Critical Group Orbits, Thesis, University of Houston, Houston. SIAM J. Math. Appl. (to appear).

Nagata, W. (1986). Unfoldings of degenerate Hopf bifurcations with $O(2)$ symmetry. Dyn. Stab. Sys. 1, 125-158.

Rand, D. (1982). Dynamics and symmetry: Predictions for modulated waves in rotating fluids. Arch. Rational Mech. Anal. 79, 1-38.

Renardy, M. (1982). Bifurcation from rotating waves. Arch. Rational Mech. Anal. 75, 49-84.

Ruelle, D. (1973). Bifurcations in the presence of a symmetry group. Arch. Ration. Mech. Anal. 51, 136-152.

Sacker, R. J. (1964). On invariant surfaces and bifurcation of periodic solutions of ordinary differential equations. Tech. Rep. IMM-NYU, New York University.

Swift, J. W. (1984). Bifurcation and Symmetry in Convection, Thesis, University of California, Berkeley.

Swift, J. W. (1988). Hopf bifurcation with the symmetry of the square. Nonlinearity 1, $333-377$.

Takens, F. (1974). Singularities of vector fields. Publ. Math. I.H.E.S. 43, 47-100.

Vanderbauwhede, A., Krupa, M., and Golubitsky, M. (1988). Secondary bifurcations in symmetric systems. In Dafernos, C. M., Ladas, G., and Papanicolaou, G. (eds.), Differential Equations: Proceedings of the EQUADIFF Conference, Marcel Dekker, Inc., New York, 1989, pp. 709-716.

van Gils, S. A., and Mallet-Paret, J. (1986). Hopf bifurcation and symmetry: standing and travelling waves on a circle. Proc. Roy. Soc. Edinburgh 104A, 279-307.

van Gils, S. A., and Valkering, T. (1986). Hopf bifurcation and symmetry: standing and travelling waves in a circular chain. Japan. J. Appl. Math. 3, 207-222.

van Gils, S. A., Iooss, G., and Los, J. (1989). On the persistence of 3-tori in the 1:1 resonance (in preparation). 